\title{
FAULT ISOLATION FOR AN INDUSTRIAL GAS TURBINE WITH A MODEL-BASED DIAGNOSIS APPROACH
}

\author{
Emil Larsson,, Jan Åslund, Erik Frisk, Lars Eriksson \\ Division of Vehicular Systems \\ Department of Electrical Engineering \\ Linköping University \\ Linköping, SE-581 83 \\ Sweden \\ Email: \{lime, jaasl, frisk, larer\}@isy.liu.se
}

\begin{abstract}
Model based diagnosis and supervision of industrial gas turbines are studied. Monitoring of an industrial gas turbine is important as it gives valuable information for the customer about service performance and process health. The overall objective of the paper is to develop a systematic procedure for modelling and design of a model based diagnosis system, where each step in the process can be automated and implemented using available software tools. A new Modelica gas media library is developed, resulting in a significant model size reduction compared to if standard Modelica components are used. A systematic method is developed that, based on the diagnosis model, extracts relevant parts of the model and transforms it into a form suitable for standard observer design techniques. This method involves techniques from simulation of DAE models and a model reduction step. The size of the final diagnosis model is $20 \%$ of the original model size. Combining the modeling results with fault isolation techniques, simultaneous isolation of sensor faults and fault tolerant health parameter estimation is achieved.
\end{abstract}

\section{INTRODUCTION}

Monitoring of an industrial gas turbine is important as it gives valuable information for the customer about service performance and process health. The goal of the monitoring system is to supervise components in the process like sensors, actuators, compressors and turbine. Degradation of the gas turbine health is

\footnotetext{
*Address all correspondence to this author.
}

typically described by health parameters, see for example [1-3]. Such health parameters are correcting factors for mass flow capacity and efficiency in compressors, turbines, nozzles, etc.

The overall objective of the paper is to develop a systematic procedure for modelling and design of a model based diagnosis system, where each step in the process can be automated and implemented using available software tools.

With a given model, fundamental questions are: Is it at all possible to detect and isolate faults of interest and how can signal processing algorithms be designed to detect and isolate the faults? How can the model be transformed into a form suitable for standard fault detection and isolation techniques? How can a Modelica model, [4], developed for simulation purposes, be used to obtain a diagnosis and process supervision system?

\section{GAS TURBINE MODELS}

Two different models are used in this paper. The first is a simulation platform that has been provided by Siemens Industrial Turbines $\mathrm{AB}$ and includes a gas turbine model as well as its surrounding systems. This model will be referred to as the reference model. The other is a gas turbine model that is of reduced order and has been tailored for diagnosis applications and will be referred to as the diagnosis model.

\section{Reference Model and Simulation Platform}

The available simulation platform provides a full featured real world example of an industrial gas turbine with its support
Copyright (c) 2010 by ASME 


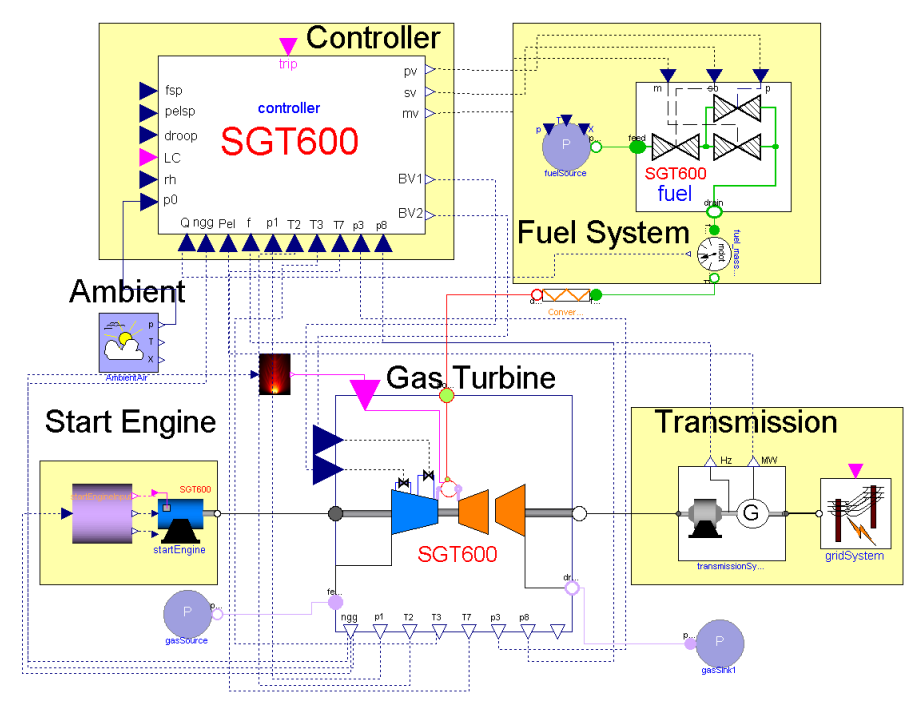

FIGURE 1. SIMULATION PLATFORM FOR SGT MODEL.

systems. Fig. 1 shows an overview of the complete system that consists of a two shaft gas turbine, a controller, a starter engine, a fuel system and a transmission system.

The platform can, for example, be used to simulate a startand stop trip and other dynamic operational cases. The diagnosis model need to capture only the behavior of the system where diagnosis is performed. Here, we consider diagnosis when bleed valves BV1 and BV2 are closed and the gas turbine is working in stationary operation.

The complete model introduced above will be used in the evaluation of the diagnosis system, involving complete system simulation and realistic trajectories. More details about the model can be found in [5].

\section{A Reduced Gas Media Package}

One step in the design of a diagnosis system and its implementation is the handling of computational complexity. Modelica provides libraries and gas components which makes it easy to build very detailed models of gases, and a key part of a thermodynamic model is the involved media package. This package is very general, since different types of thermodynamic process can be modeled. An ideal gas can for example be described by a gas mixture with a number of species. A gas volume component that is provided by the Modelica standard library has a mass state for each species in the mixture, and when the number of volume components and gas mixture species in a model increases, the number of equations increases drastically. As an example, the reference SGT model has about 2500 equations. However for the implementation of a diagnosis system this level of detail can give an unnecessarily high computational burden. With simple means this drawback can be amended and a method that achieves this will be developed below.

One key step for reducing the model lies in the formulation of the equations for the gas medium. The most important step is to lump the description of gas molecules together and use a single mixture strength parameter to describe the essential gas property. To exemplify this we can look at air, that consists of $\mathrm{N}_{2}, \mathrm{O}_{2}, \mathrm{CO}_{2}, \mathrm{H}_{2} \mathrm{O}$, Ar, and other molecules. These species can be lumped into a single component called Air. This is the point where the main reduction in states occur and a simpler, equivalent, description is obtained under the assumption that the gases are fuel and air. Further simplifications in the equations can be achieved by utilizing knowledge that the gas turbine always operates at fuel lean conditions. With these assumptions we can use the oxygen (or pure air) in the burned gases as a state, see [6,7] for examples where this is done in internal combustion engines. To describe this, the relative air/fuel ratio $\lambda[8]$ is used as a state for burned gases. To specify the state of the gas in the reduced media package, it is necessary to give the pressure, temperature and air/fuel ratio.

Combustion Stoichiometry. In the next step the equations that govern the state variable $\lambda$ will be developed and it is the combustion process that has a main influence here. The hydrocarbon fuel considered in the reference model is discussed in [5], and has the following chemical reaction formula:

$$
n_{a} \tilde{x}_{a}+n_{f} \tilde{S} \tilde{x}_{f} \rightarrow n_{b} \tilde{x}_{b}
$$

where $n_{i}$ is the number of mole and $\tilde{S}$ is the stoichiometry matrix

$$
\tilde{S}=\left(\begin{array}{ccccc}
0 & 0 & 0 & 0 & 0 \\
2 & 3 & 1 & 1 & 0 \\
3 & 4 & 2 & 0 & 0 \\
0 & 0 & 0 & 0 & 1 \\
-3.5 & -5 & -2 & 0 & 0
\end{array}\right)
$$

$\tilde{x}_{a}, \tilde{x}_{f}$ and $\tilde{x}_{b}$ are the given mole concentrations of air, fuel and burned gases as follow:

$$
\tilde{x}_{a}=\left(\begin{array}{c}
\tilde{x}_{a, A r} \\
\tilde{x}_{a, C O_{2}} \\
\tilde{x}_{a, H_{2} O} \\
\tilde{x}_{a, N_{2}} \\
\tilde{x}_{a, O_{2}}
\end{array}\right), \tilde{x}_{f}=\left(\begin{array}{c}
\tilde{x}_{f, A r} \\
\tilde{x}_{f, C O_{2}} \\
\tilde{x}_{f, H_{2} O} \\
\tilde{x}_{f, N_{2}} \\
\tilde{x}_{f, O_{2}}
\end{array}\right), \tilde{x}_{b}=\left(\begin{array}{c}
\tilde{x}_{b, A r} \\
\tilde{x}_{b, C O_{2}} \\
\tilde{x}_{b, H_{2} O} \\
\tilde{x}_{b, N_{2}} \\
\tilde{x}_{b, O_{2}}
\end{array}\right) .
$$

If mass concentrations are considered, the stoichiometric air/fuel ratio is:

$$
\left(\frac{m_{a}}{m_{f}}\right)_{s}=\frac{3.5 \frac{x_{f, C_{2} H_{6}}}{M_{C_{2} H_{6}}}+5 \frac{x_{f, C_{3} H_{8}}}{M_{C_{3} H_{8}}}+2 \frac{x_{f, C H_{4}}}{M_{C H_{4}}}}{\frac{x_{a, O_{2}}}{M_{O_{2}}}} \equiv(A / F)_{s}
$$

where $M_{i}$ is molecular weights of species $i$, and $x_{k, l}$ is mass concentration of gas $k$ and species $l$. Indices $S$ is used to denote that 
air/fuel ratio is expressed in masses. The definition of the relative air/fuel ratio $\lambda[8]$ is:

$$
\lambda=\frac{m_{a} / m_{f}}{(A / F)_{s}}
$$

If the combustion is lean, i.e., $\lambda>1$, the mass concentration of burned gases are:

$$
X(\lambda)=\frac{m_{b} x_{b}}{m_{b} \sum_{i} x_{b, i}}=\frac{m_{a} x_{a}+m_{f} S x_{f}}{m_{a} \sum_{i} x_{a, i}+m_{f} S x_{f, i}}=\frac{(A / F)_{s} \lambda x_{a}+S x_{f}}{(A / F)_{s} \lambda+1}
$$

where lambda definition (5) and reaction formula (1), expressed in mass fractions are used. Since masses are conserved, the normalization factor is simply equal to $(A / F){ }_{s} \lambda+1$.

Perfect Mixing in a Control Volume. If all gases in a volume are perfectly mixed, the change in mass fraction is consequently described by the following differential equation:

$$
\frac{d X(\lambda(t))}{d t}=\frac{m_{\text {flow,in }}(t)}{m(t)}\left(X\left(\lambda_{\text {in }}(t)\right)-X(\lambda(t))\right)
$$

where $X(\lambda(t))$ describes, as a function of $\lambda$, species concentration of the gas in the volume. Incoming mass flow, concentration of incoming gas and total mass in the volume are $m_{\text {flow,in }}, \lambda_{\text {in }}$ and $m$ respectively. Differential equation (7) can be expressed in $\lambda$ only:

$$
\frac{d \lambda}{d t}=\frac{m_{\text {flow, in }}}{m} \cdot \frac{(A / F)_{s} \lambda+1}{(A / F)_{s} \lambda_{\text {in }}+1}\left(\lambda_{\text {in }}-\lambda\right)
$$

if (4), (6) and (7) are used. If more than one incoming mass flow are considered, (8) has to be replaced with a sum over all $\lambda_{i n}$. Note: it is only positive mass flows that influence the concentration $\lambda$.

Combustion Temperature. For combustion it is assumed an adiabatic constant-pressure combustion process. Then it holds that enthalpy $h_{u}\left(T_{1}\right)$ before combustion and enthalpy $h_{b}\left(T_{2}\right)$ after combustion are equal [8]. If $h_{b}\left(T_{2}\right)$ is invertible, it is possible to find a solution: $T_{2}=h_{b}^{-1}\left(h_{u}\left(T_{1}\right)\right)$, which is the combustion temperature. If the inverse isn't available then a numerical equation solver can be applied without loss of generality.

\section{Gas Turbine Components Library - GTlib. mo}

In the next step the most important components are presented. These components are included in the gas turbine library GTlib. mo and are used in the reduced SGT model. These components are similar to those in the reference model with the main difference related to the usage of $\lambda$ for the state instead of individual species components.
Volume. The volume component has the following states:

$$
\begin{aligned}
\frac{d m}{d t} & =\sum_{i} m_{\text {flow }, i} \\
\frac{d E_{\text {tot }}}{d t} & =\sum_{i} H_{\text {flow }, i} \\
\frac{d \lambda}{d t} & =\sum_{i} \frac{m_{\text {flow }, \text { in }, i}}{m} \cdot \frac{(A / F)_{s} \lambda+1}{(A / F)_{s} \lambda_{\text {in }, i}+1}\left(\lambda_{\text {in }, i}-\lambda\right)
\end{aligned}
$$

where $\sum m_{\text {flow }}$ is the total mass flow, and $\sum H_{\text {flow }}$ is the total energy flow into the volume. Note that in $(9 \mathrm{c})$, only positive mass flows are considered. The mass $m$ and total energy $E_{t o t}$ are simply: $m=V d$ and $E_{t o t}=m u$, where $V, d$ and $u$ are volume, density and internal energy respectively.

Orifice. The orifice is a simple pressure loss component where no mass or energy are accumulated. The pressure drop is described by: $m_{\text {flow }}=C \sqrt{p_{1}-p_{2}}$ where $C$ is a model specific constant and $p_{1}, p_{2}$ are pressure upstream and downstream.

Shaft. In Modelica is the shaft angle $\varphi$, instead of angular velocity $\omega$, is used as a connection variable between mechanical components. This results in an additional angle $\varphi$ state as:

$$
\begin{aligned}
\dot{\varphi} & =\omega \\
J \dot{\omega} & =\Delta \tau
\end{aligned}
$$

where $\Delta \tau$ is the torque difference between applied and brake torque. The angle state (10) is a pure integration of velocity if the model has no angle dependencies.

Compressor. Basic equations for the compressor component are the four functions $f_{i}$ that are used for characteristic calculations. These functions are:

$$
\begin{aligned}
\vartheta_{\text {norm }} & =f_{1}(T, R, \gamma, p) \\
\omega_{\text {norm }} & =\omega f_{2}(T, R, \gamma, p) \\
\vartheta & =\frac{1}{m_{\text {norm }}} f_{3}\left(\Pi, \omega_{\text {norm }}\right) \\
\eta_{i} & =f_{4}\left(\Pi, \vartheta \vartheta_{\text {norm }}\right)
\end{aligned}
$$

which are implemented as look-up tables. These look-up tables are developed by the manufacturer through a large number of performance tests and component simulations.

Temperature $T$, gas constant $R$, isentropic coefficient $\gamma$ and pressure $p$ are all calculated up-stream. $\Pi$ is pressure ratio, $\omega$ is rotational spool speed, and $\vartheta$ the mass flow. To calculate the 
isentropic enthalpy $h_{i s}$, the built-in functions in Modelica media package are used. These functions use the NASA polynomials described in [9]. The actual enthalpy change, through the compressor, can be calculated according to: $\Delta h=\left(h_{i s}-h\right) / \eta_{i}$, where $\eta_{i}$ is the isentropic efficiency according to (15). The connection between mechanical and thermodynamic power is:

$$
\vartheta \Delta h+\sum_{i} r_{i} \vartheta_{r_{i}}=\mu \omega \Delta \tau
$$

where $\vartheta_{r_{i}}$ is mass flow of air through bleed valve $i$ and $r_{i}$ is the given enthalpy ratio where valve $i$ is placed. The mechanical efficiency $\mu$ is assumed to be constant. Since no mass or energy is stored in the compressor, the mass and energy flows through the compressor are summed to zero.

Turbine. The turbine component is modeled in a similar manner as the compressor, and functions $g_{i}$ are used as follows:

$$
\begin{aligned}
N_{\text {norm }} & =g_{1}(R, \gamma, \tilde{T}, \Pi, \omega) \\
A_{\text {norm }} & =g_{2}(R, \gamma, \tilde{T}, \Pi, \omega) \\
A & =g_{3}\left(N_{\text {norm }}, \Pi\right) A_{\text {norm }} \\
\eta_{i} & =g_{4}\left(N_{\text {norm }}, \Pi\right)
\end{aligned}
$$

These $g_{i}$ functions are also implemented as look up tables. Here the cross section area $A$ is used instead of mass flow. Here, $\tilde{T}$ is the inlet temperature before exhaust gases are mixed with cooling air.

Combustor. The combustor component has two modes, i.e., with and without an ignition, IGN. With ignition means that burned gases are produced. Enthalpy $h_{b}$ of burned gases can be summarized according to:

$$
h_{b}= \begin{cases}h_{a} \chi_{a}+h_{f}\left(1-\chi_{a}\right), & \text { if IGN } \\ h_{a}, & \text { not IGN }\end{cases}
$$

where $\chi_{a}=\frac{m_{a}}{m_{a}+m_{f}}$ is actual mass fraction of air and $\lambda$ that is produced by the combustion is calculated according to definition (5). The mass flows of air and fuel through the combustor are described by a simple pressure drop model. Equations for conservation of mass and energy are similar to those in the earlier defined components.

\section{Diagnosis Model of the Gas Turbine}

The diagnosis model consists only of the gas turbine model, valid under stationary operating conditions when bleed-valves are closed. The gas turbine model is composed of the above defined components and is shown in Fig. 2. Primary and secondary cooling air is used to lower the temperature of hot exhaust gases to protect vanes in the first turbine stages of the high- and low pressure turbine CT and PT. This phenomenon is captured by the model, since cooling air (with a large $\lambda$ ) is mixed with exhaust gases to lower the overall gas temperature. The introduction of the reduced media package resulted in a reduction of model equations from about 2500 in the reference model to about 800 in the simplified SGT model.

Four health parameters [1-3] are introduced in the diagnosis model. Ideally, these health parameters should capture deviation from nominal value for: isentropic efficiency $\Delta \eta_{C C}, \Delta \eta_{C T}$ for CC and CT, mass flow $\Delta \vartheta_{C C}$ for CC and finally cross section area $\Delta A_{C T}$ for CT. These health parameters are interesting for process monitoring, but are also necessary to avoid false alarms when supervising the sensors [10].

The supervised health parameters can be summarized:

$$
\begin{aligned}
\eta_{C C} & =f_{4}\left(\Pi, m_{\text {flow }} m_{\text {norm }}\right)+\Delta \eta_{C C} \\
\eta_{C T} & =g_{4}\left(N_{\text {norm }}, \Pi\right)+\Delta \eta_{C T} \\
\vartheta_{C C} & =\frac{1}{m_{\text {norm }}} f_{3}\left(\Pi, \omega_{\text {norm }}\right)+\Delta \vartheta_{C C} \\
A_{C T} & =g_{3}\left(N_{\text {norm }}, \Pi\right) A_{\text {norm }}+\Delta A_{C T} \\
\Delta \dot{\eta}_{C C} & =0 \\
\Delta \dot{\eta}_{C T} & =0 \\
\Delta \dot{\vartheta}_{C C} & =0 \\
\Delta \dot{A}_{C T} & =0
\end{aligned}
$$

where actual performance equations are extended with the deviation parameter. Since the performance variables are assumed to vary slowly, due to natural performance degradation over time, the derivatives (26)-(29) are set to zero in the nominal model.

Sensors and Actuators. The diagnosis model has 2 actuator and 8 output signals. These signals are measured pressures, temperatures and rotational speeds. Known actuator signals are fuel pressure and demanded torque from the generator, which are inputs to the diagnosis model delivered by the environment. Ignition signal IGN is always true, and is therefore not considered as an actuator signal. Where measurement sensors are placed can be seen in Fig. 2.

The physical gas turbine is equipped with the same set of sensors as in the model. In the transmission system power and angular velocity of the PT shaft are measured so it is possible to calculate the demanded torque. Further is the mass flow of fuel measured in the gas turbine but in the model is fuel pressure used.

Measurement Noise. Band-limited white noise is added to all known signals. The added noise has a Gaussian amplitude distribution with a standard deviation, in percent, in the range of $0.1-0.5$ of the nominal value for each measured quantity, see Tab. 1. Sensors $y_{5}$ and $y_{7}$ measure, more or less, the ambient 


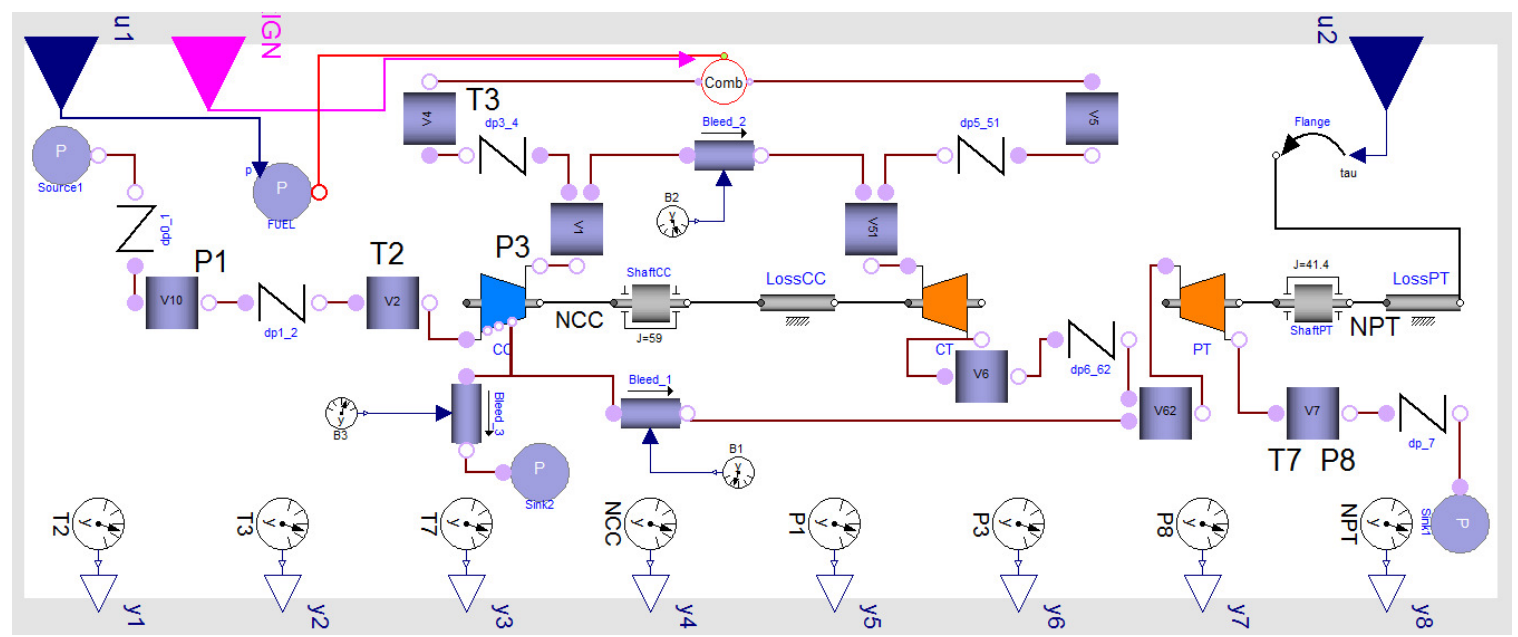

FIGURE 2. REDUCED SGT GAS TURBINE MODEL USED FOR DIAGNOSIS.

TABLE 1. SENSOR AND ACTUATOR NOISE STANDARD DEVIATION IN PERCENT OF THE NOMINAL VALUES.

\begin{tabular}{lcl}
\hline Sensor & Quantity & $\sigma$ \\
\hline \hline$y_{5}, y_{7}$ & P1, P8 & $0.1 \%$ \\
$y_{1}, y_{2}, y_{3}, y_{6}$ & T2, T3, T7, P3 & $0.5 \%$ \\
$y_{4}, y_{8}$ & NCC, NPT & $0.15 \%$ \\
\hline Actuator & Quantity & $\sigma$ \\
\hline \hline$u_{1}, u_{2}$ & P10, TT & $0.4 \%$ \\
\hline
\end{tabular}

pressure and are therefore assumed to be more reliable then the high pressure sensor $y_{6}$. It is also assumed that the rotational sensors $y_{4}$ and $y_{8}$ are reliable and therefore have smaller noise amplitude than the sensors $y_{1}, y_{2}, y_{3}$ and $y_{6}$.

Faults. Faults that are diagnosed in this paper are faults in measurement sensors viewed in Tab.1. The considered faults are modeled as additive signals,

$$
y_{i}=x_{i}+f_{i}
$$

for sensor $i$ where $x_{i}$ is the measured quantity and $y_{i}$ the measurement signal.

\section{DIAGNOSABILITY ANALYSIS OF THE MODEL}

The model of the gas turbine, developed in the previous section, included descriptions of faults in 8 sensors and inclusion of 4 health parameters capturing drift in important performance parameters.

Before a diagnosis and supervision system is designed, a fundamental question is what diagnosability performance is at all possible, i.e., are faults and changes in parameters in (22)-(25) and (30) detectable and isolable? Without becoming formal, a fault is said to be detectable in a system if there exists operating conditions such that the observations deviate from what is expected from a healthy gas turbine. Note that detectability is dependent on the particular sensor configuration used. Isolability among faults can be defined in a similar manner, a fault $f_{1}$ is isolable from a fault $f_{2}$ if there exists operating conditions such that the observations are consistent with fault mode $f_{1}$ but not with fault $f_{2}$.

Unfortunately, to obtain exact answers to questions concerning detectability and isolability, exact characterization of solutions to the model equations are needed. In general, this is not feasible unless the model is for example linear. See e.g. [11] for formal definitions and how to compute isolability in the linear case. The model developed in this paper is a non-linear, differentialalgebraic, model where no such characterization is possible. Instead, an approach that only utilizes the model structure is used. The structure is a coarse description of the model where for each model equation, it is noted which model variables that are included in the equation. Fig. 3 show the model structure for the gas turbine where each blue dot indicates that the variable is included in the corresponding model equation.

In [12], such coarse model information is used to efficiently determine detectability and isolability properties of the model. Since only the structure and not the analytical expressions is used, only best-case results are obtained.

The variables and equations have been permuted such that Fig. 3 reveals detectability and isolability properties of the model. This form can be obtained efficiently and fast for large models. For precise details on how to obtain this form, see $[12,13]$. The main computational step utilizes the Dulmage-Mendelsohn de- 


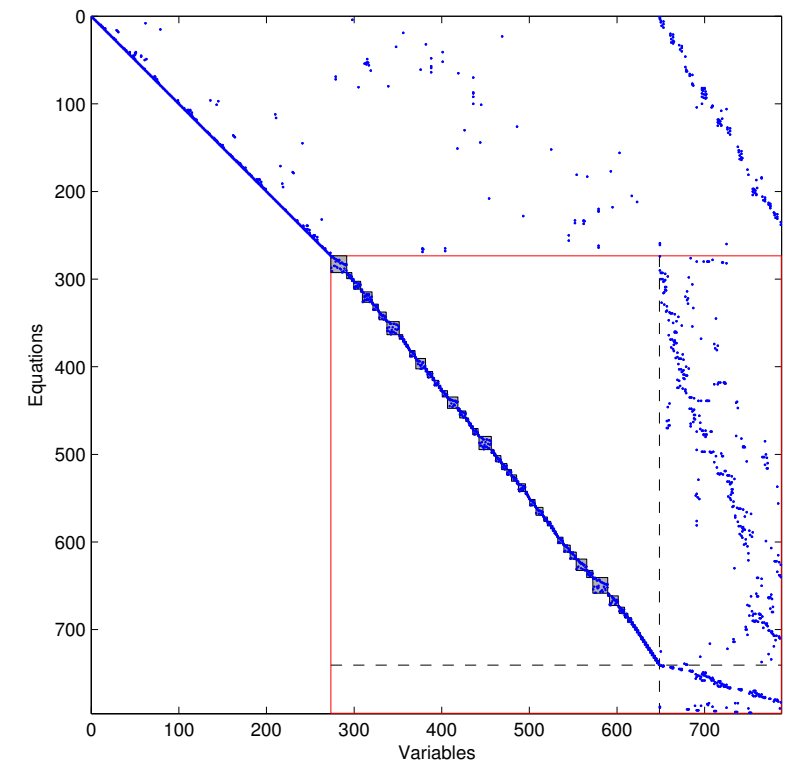

FIGURE 3. STRUCTURE OF THE GAS TURBINE MODEL.

composition of graphs [14]. The first 250 equations belong to the exactly determined part, i.e., parts where there are no redundancy and thus any fault affecting this part of the system can not be detected. The overdetermined part of the system that contains redundancy is indicated by the large rectangle, and thus any faults affecting this part are detectable. The figure also reveals the isolability properties of the system. For example the small gray boxes indicate sets of model equations that can not be isolated. This means that if two faults appear in two equations with variables in the same gray box, the faults can not be isolated.

Looking at the particular model described in Section 2, all the considered faults belong to the overdetermined part, i.e., all faults are detectable. It can also be directly verified that all faults appear in equations with no variables in the same gray box and it is therefore theoretically possible to detect and isolate the faults. However, since the model is non-linear it may be the case that it can be difficult to design a diagnosis system that can reach what is theoretically possible.

\section{METHOD DESCRIPTION}

The objective, as stated in the introduction, is fault tolerant estimation of the health parameters and simultaneous diagnosis of faults in the sensors $\left\{y_{1}, \ldots y_{m}\right\}$. The method is based on a bank of observers that estimate the health status of the gas turbine and a diagnoser that detects faults in the sensors and chooses the appropriate health status estimator depending on the current diagnosis. This means that as long as the diagnoser delivers correct information to the estimator selector, reliable health status estimation is given in spite of faults in the sensors. One fundamental problem is that the diagnoser, in the case of a sensor fault, may not be able to exactly determine which is the faulty sensor, but may only be able to determine that one in a set of sensors is faulty. How to deal with this is treated later, but first a description of the detection and isolation procedure.

The fault detection and isolation logic is based on hypothesis testing and there is one hypothesis for each sensor failure. Such an approach is standard in general diagnosis methodology [14] and has been used for supervision of gas turbines in, e.g., [3, 15-17]. Let $H_{i}$ denote the hypothesis that sensor $y_{i}$ is faulty or that there are no faulty sensors. For each fault hypothesis $H_{i}$, a constant gain Kalman filter is designed where all except the supervised sensor $y_{i}$ are used for feedback in the observer. Full details on observer design are given in the next section. Now, let $Y_{i}$ denote the vector that excludes sensor measurement $y_{i}$, i.e., $Y_{i}=$ $\left(y_{1}, \ldots, y_{i-1}, y_{i+1}, \ldots, y_{m}\right)^{T}$. Then for each hypothesis, an alarm is generated if the estimated output deviates significantly from the measured output. Each filter computes the output estimation error vector $e_{i}$ as

$$
e_{i}(t)=\hat{Y}_{i}(t)-Y_{i}(t)
$$

where $\hat{Y}_{i}$ is the estimate of $Y_{i}$. In case that hypothesis $H_{i}$ is true, it is expected that all estimation errors, except $e_{i}$, will become large, since they all use the faulty sensor $y_{i}$ for feedback in the filter. The weighted sum of squared estimation errors

$$
R_{i}(t)=e_{i}^{T}(t) \Sigma_{i}^{-1} e_{i}^{T}(t), \quad \Sigma_{i}=\operatorname{diag}\left[\sigma_{j}^{2}\right]_{j \neq i}
$$

is computed, where $\sigma_{j}$ is the standard deviation for sensor $y_{j}$, and if $R_{i}$ significantly deviates from zero, an alarm is generated. A simple and effective way to determine a significant deviation of $R_{i}(t)$ from zero is to apply the CUSUM algorithm [18] where the quantity $T_{i}(t)$ is computed as

$$
T_{i}(t)=\max \left(0, T_{i}(t-1)+\left|R_{i}(t)\right|-v_{i}\right), \quad T_{i}(0)=0
$$

and an alarm is generated when $T_{i}(t)$ exceeds a specified threshold. The parameter $v_{i}$ is a design parameter for the algorithm designer to choose and a rule of thumb is that $v$ is of the same order of magnitude as the size of $R_{i}(t)$ in the fault free case.

To summarize, each observer is insensitive to a fault in the corresponding sensor $y_{i}$ but sensitive to faults in all other sensors which means that the faulty sensor can be isolated by observing which hypothesis whose test triggers an alarm.

In addition to the fault hypothesis, the no fault hypothesis $H_{N F}$ is included in the framework and all sensors are used as feedback in the corresponding Kalman filter. Thus, if there is no alarm, the health parameter estimates are taken from the observer which utilizes feed-back from all sensor signals. In case of an alarm and a unique isolation of a faulty sensor, the filter for which 
the hypothesis is not rejected is used to obtain estimates of the health parameters.

In an ideal case, where the model perfectly describes the real system and where there is no measurement noise, it would be sufficient to consider the test quantities $T_{i}(t)$ to detect and isolate single faults. A single fault would result in all hypothesis except the correct one being rejected. However, due to uncertainties and noise some of the test quantities, that ideally should trigger an alarm, do not alarm. This means that a fault is detected but there is not a unique diagnosis, two or more candidates for faulty sensors are produced by the fault isolation logic and we do not know exactly which health parameter estimates to use. What we need then is sometimes referred to as focusing of the diagnoses, i.e., an estimation of the most likely sensor to have failed. For that reason, a second type of residual is introduced as a complement to the test described above:

$$
r_{i}=\left|\hat{y}_{i}-y_{i}\right| / \sigma_{i}
$$

where $\hat{y}_{i}$ is the estimate of $y_{i}$ obtained using the filter corresponding to hypothesis $H_{i}$. A property of this residual is that it is sensitive to all sensor faults since all but senor $y_{i}$ is used in the estimation of $\hat{y}_{i}$ and sensor $y_{i}$ is used when computing residual $r_{i}$. In addition, the residual $r_{i}$ can be expected to be more sensitive to a fault in sensor $y_{i}$ than those in vector $Y_{i}$ since the latter set of sensors are fed back in the observer. If there is more than one candidate diagnosis, a simple approach to choose the most likely is to evaluate which of the corresponding $r_{i}$ has the strongest response.

The method can now be summarized as:

1. Compute $T_{i}(t), i=1, \ldots, m$ according to (33).

2 . If no test triggers an alarm, obtain the health parameter estimates from an observer using feedback from all sensors.

3. In case of an alarm, candidate diagnoses are those sensors that correspond to $T_{i}(t)$ that have not exceeded its threshold. If only one test quantity $T_{i_{\text {fault }}}(t)$ is below its corresponding threshold, a unique isolation has been achieved. In case the isolation is not unique, let $i_{\text {fault }}$ correspond to the case where $r_{i}$ in (34) has the most significant response.

4. Obtain the health parameter estimates from the observer corresponding to hypothesis $H_{i_{\text {fault }}}$

\section{OBSERVER DESIGN}

The observer design problem is to take the Modelica model and automatically design an observer, estimating the state and health parameters. Since the diagnosis model is in a general differential algebraic equation (DAE) form, standard observer design techniques are not directly applicable. Therefore, a number of model transformations are needed, and then a standard Kalman filter design will be used.

The flat form of the Modelica representation of the diagnosis model, where the measurement equations are excluded, is in the form

$$
\begin{aligned}
E \dot{x}_{1}^{0} & =f^{0}\left(x_{1}^{0}, x_{2}^{0}, u\right) \\
0 & =g^{0}\left(x_{1}^{0}, x_{2}^{0}, u\right)
\end{aligned}
$$

where $E$ is a constant matrix, $u$ are known actuator signals, $x_{1}^{0}$ are the dynamic variables, including the health parameters $h$, and $x_{2}^{0}$ are the algebraic variables. Thus, the non-linear function $f^{0} \mathrm{de}$ scribes the dynamic behavior and $g^{0}$ are the algebraic constraints in the model.

\section{DAE Index Analysis}

A model with a DAE differential index 0 or 1 is generally easier to handle in practise than a system with a higher index [19]. Index analysis of (35) shows that the DAE has differential index equal to 2 and that it is possible to reduce the index if some of the equations are differentiated. Pantelides algorithm [20] is used to determine which equations that need to be differentiated.

To explain the high index property of the model, observe that in the Modelica standard library, angle dependencies are used in the equations that connect mechanical rotation components. These equations have the form: $\varphi_{1}=\varphi_{2}, \varphi_{2}=\varphi_{3}, \ldots$ and Pantelides algorithm differentiate these equations to obtain, $\dot{\varphi}_{1}=\dot{\varphi}_{2}, \dot{\varphi}_{2}=$ $\dot{\varphi}_{3}$, and so on. Extending the model with these differentiated equations yields a DAE with differential index 1 .

\section{Semi-explicit DAE}

The next step is to obtain a semi explicit form:

$$
\begin{aligned}
\dot{x}_{1}^{1} & =f^{1}\left(x_{1}^{1}, x_{2}^{1}, u\right) \\
0 & =g^{1}\left(x_{1}^{1}, x_{2}^{1}, u\right)
\end{aligned}
$$

This is done by simple algebraic manipulations of the model equations, i.e., QR decomposition of $E$. For example, the relations $\omega_{1}=\dot{\varphi}_{1}$ and $\omega_{2}=\dot{\varphi}_{2}$ are substituted into the dynamic equation $\dot{\varphi}_{1}=\dot{\varphi}_{2}$ to obtain the static equation $\omega_{1}=\omega_{2}$, which is now part of the algebraic constraint $g^{1}=0$. Now, a DAE with index of 1 means that the Jacobian $\partial g^{1} / \partial x_{2}^{1}$ is non-singular.

\section{Model Reduction}

In this step, parts of the model are removed that are not relevant for a particular observer design problem. For example, consider the Kalman filter for hypothesis $H_{i}$. Since the measurements in $Y_{i}$ are used in the filter, it is necessary that the corresponding variables are included in the model. Furthermore, since the signal $y_{i}=x_{i}$ is used in the residual (34), this variable should also be included in the model.

The objective is to transform the model into a set of ordinary differential equations. This will be done by eliminating the 
algebraic variables using a numerical solver, and it therefore necessary that the number of algebraic equations and the number of algebraic variables are equal. Note that the number of dynamic variables and the number of dynamic equations in (36a) are equal by construction.

To summarize the discussion, a submodel is sought that includes all the measured variables, and where there are as many equations as unknowns. One way to find the smallest subset that fulfills these requirements is to first add the sensor equations $y_{i}=x_{i}$ to the model and then compute the overdetermined part of the model, which was introduced in Section 3. By removing the measurement equations from the set of equations obtained by this operation, the sought set is obtained. This follows from the theory developed in [12].

After this operation, about 500 equations remain in the model which is in the form

$$
\begin{aligned}
\dot{x}_{1}^{2} & =f^{2}\left(x_{1}, x_{2}, u\right) \\
0 & =g^{2}\left(x_{1}, x_{2}, u\right)
\end{aligned}
$$

For example, the dynamic equations $\dot{\varphi}_{i}=\omega_{i}$ are removed in this step of the design procedure. This indicates that the values of angles $\varphi_{i}$, which are computed by integrating these equations, are not needed in the diagnostic system.

\section{State-Space Form}

The main objective with the series of model transformations is to obtain a standard state-space description and now a final step finalizes this transformation. It can be shown that the DAE index is also 1 after the submodel is extracted from the original model. Hence, the the Jacobian matrix $\partial g^{2} / \partial x_{2}$ is non-singular and it is possible, at least locally, to use a numerical solver to compute $x_{2}=$ $G\left(x_{1}, u\right)$ from the algebraic condition $0=g^{2}\left(x_{1}, x_{2}, u\right)$. Insertion of $x_{2}$ into $f^{2}$ then gives the model

$$
\dot{x}_{1}=f\left(x_{1}, u\right)
$$

where $f\left(x_{1}, u\right)=f^{2}\left(x_{1}, G\left(x_{1}, u\right), u\right)$ which is the desired ODE form.

\section{Observer Design}

Now that the system has been transformed into a state-space description, standard observer design techniques can be utilized. A possible first choice is an Extended Kalman Filter but here a simpler approach is adopted. Let $Y_{i}=h_{i}\left(x_{1}\right)$ be the measurement equation, then the non-linear model is numerically linearized in a suitable operating point to obtain a linear approximation of the system. A standard, stationary, Kalman filter design is then performed to obtain a constant feedback gain $K_{i}$. The non-linear observer is then formed as

$$
\dot{\hat{x}}_{1}=f\left(\hat{x}_{1}, u\right)+K_{i}\left(Y_{i}-h_{i}\left(\hat{x}_{1}\right)\right)
$$

which provides an estimate of the dynamic variables $x_{1}$, which includes the health parameters, based on the measurement signals in $Y_{i}$. It is straightforward to extend the design with linearizations in more than one operating point together with a gain switching procedure.

\section{SIMULATION RESULTS}

The diagnosis system consists of 9 observers that are automatically generated in Matlab for simulation in a Modelica simulation tool. Design parameters for the observers are the noise covariance matrices $Q$ and $R$, corresponding to process and measurement noise. The observers are tuned such that the dynamics for the health parameters are much slower than the dynamics for other state variables.

\section{Experiment Setup}

Data for the diagnosis system are collected from the full-scale simulation platform where the SGT gas turbine model, viewed in Fig.1, is used. The simulation study is a start-and-stop trip and data are collected when the gas turbine reaches the operational point. A sensor fault is modeled as a bias term that is added to the sensor signal.

Degradation in performance parameters, with a certain profile, are inserted in the actual performance parameter in the SGT gas turbine model. The simulated degradation in efficiency $\eta_{i}$ is 0.03 for CC and 0.02 for CT. Degradation in mass flow $\vartheta_{C C}$ and area $A_{C T}$ are 0 in both cases. See Fig. 6.

\section{Test Case - Sensor Fault in Temperature Sensor $y_{2}$}

To demonstrate fault detection a sensor fault is considered here. In this case, a constant bias term with an amplitude of 5\% of the fault free value is added to sensor $y_{2}$ at time $14 \mathrm{~h}$.

The residual for four of the hypothesis can be seen in Fig. 4. It can be seen a response in $R_{1}, R_{6}$, and $R_{8}$, as expected, and none in $R_{2}$. The remaining residuals, omitted due to space limitations, show a similar behavior. Since the residuals are affected by noise, the CUSUM algorithm is applied to obtain more reliable test quantities, which are shown in Fig. 5. The only test quantity that does not trigger an alarm by the fault is $T_{2}$. Hence, the fault is correctly detected and isolated.

Now, it can be concluded that hypothesis $\mathrm{H}_{2}$ is the correct one, and the corresponding observer can be used as health parameter estimator, see Fig. 6, after the fault has been detected. Before detection of the fault, the health parameter estimates are provided by the observer where all measurements are used.

The difference between the faulty sensor and estimated temperature T3 of observer 2 can be seen in Fig. 7. Here a strong response can be seen, which means that the fault will be clearly visible in the residual $r_{2}$ defined in (34).

To summarize the discussion, these simulations show how the proposed method can simultaneously isolate sensor faults and estimate health parameters. 

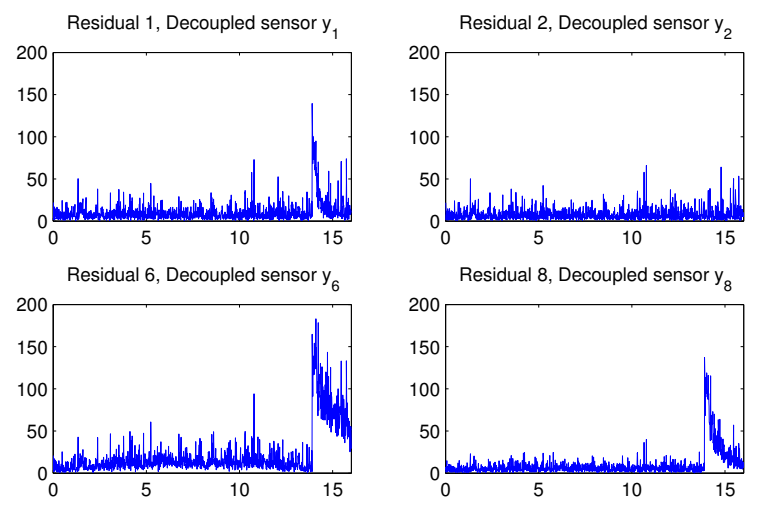

FIGURE 4. RESIDUALS FOR DECOUPLED SENSOR $y_{1}, y_{2}, y_{6}$ and $y_{8}$ WITH A FAULTY SENSOR $y_{2}$.
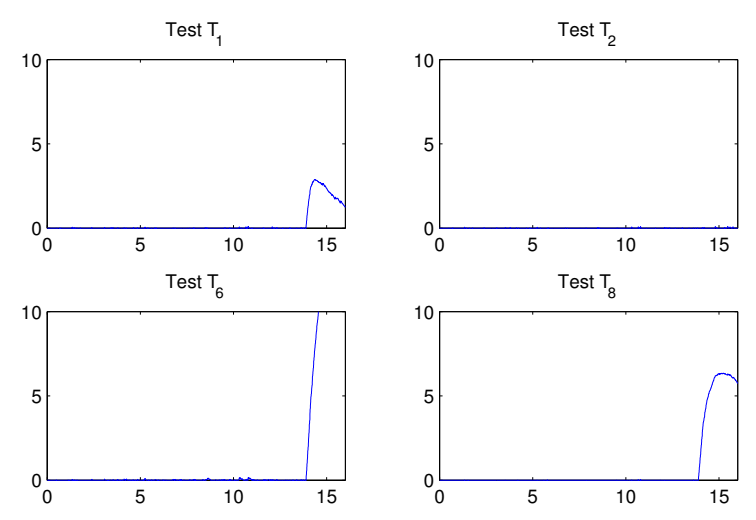

FIGURE 5. CUSUM APPLIED TO THE RESIDUALS IN FIG. 4.

\section{CONCLUSIONS}

A gas turbine model suitable for diagnosis analysis and design has been developed. The diagnosis model is based on a Modelica model developed for simulation purposes, where the standard Modelica library is used. The diagnosis model is equivalent, but has a significantly reduced number of equations. This reduction was accomplished by development of a new media library. For the gas turbine considered here, the reduction is from 2500 to 800 equations.

The second main contribution is a systematic method that, based on the diagnosis model, extracts relevant parts of the model and transforms it into a form suitable for standard observer design techniques. This method involves standard techniques from simulation of DAE models and another model reduction step, where the number of equations are reduced to about 500. Combining these methods with standard fault isolation techniques, simultaneous isolation of sensor faults and fault tolerant health parameter estimation is achieved.
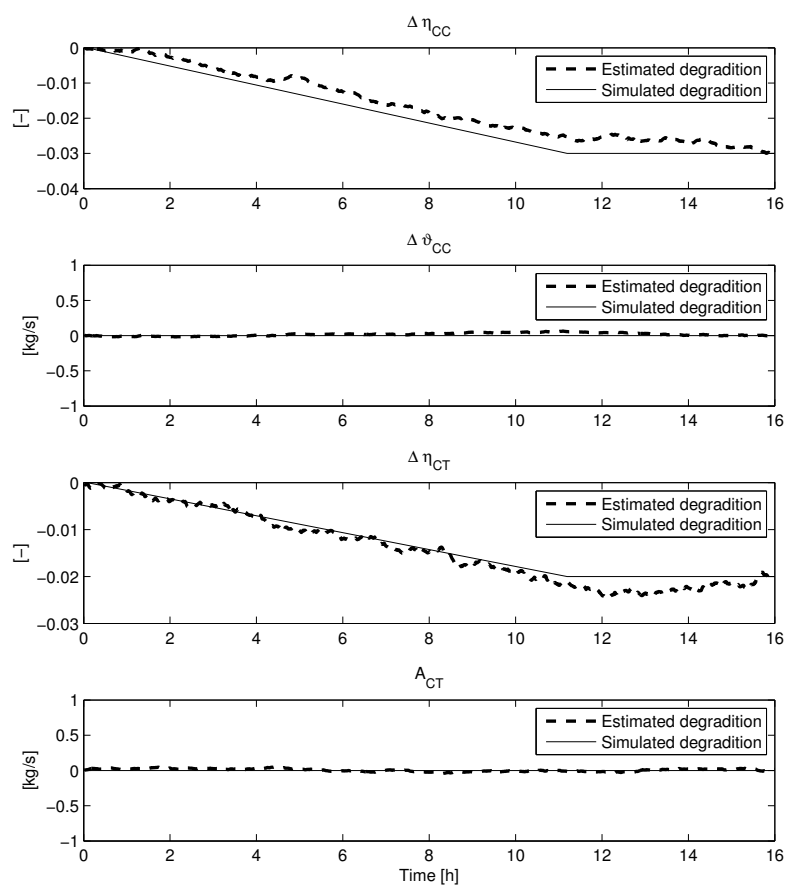

FIGURE 6. HEALTH PARAMETERS FOR OBSERVER 2 - DECOUPLED SENSOR $y_{2}$.

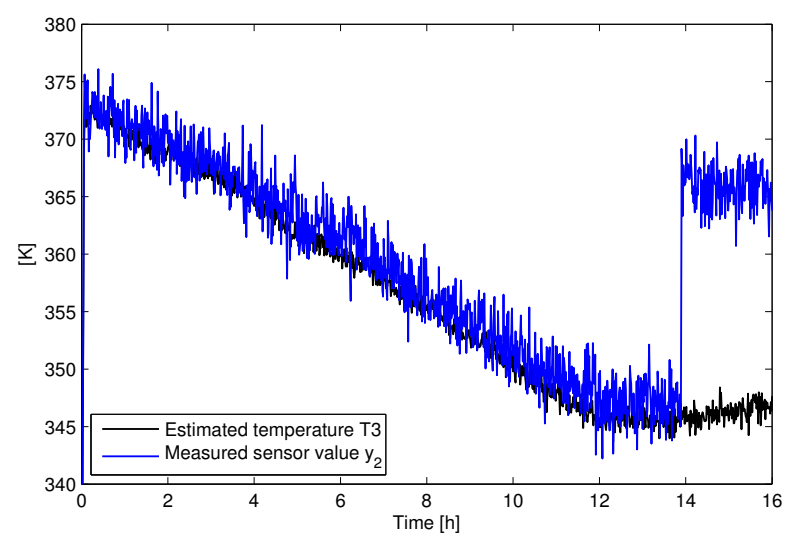

FIGURE 7. ESTIMATED T3 AND FAULTY SENSOR $y_{2}$.

The method has been successfully evaluated in a simulation study, where a fault is introduced in a temperature sensor while the health parameters for isentropic efficiency and mass flows in the compressor are degraded. 


$\begin{array}{ll}\text { NOMENCLATURE } \\ \text { CC } & \text { Compressor } \\ \text { CT } & \text { High-pressure turbine } \\ \text { PT } & \text { Low-pressure turbine } \\ \text { P1 } & \text { Compressor inlet static pressure, measured } \\ \text { T2 } & \text { Compressor inlet total temperature, measured } \\ \text { P3 } & \text { Compressor outlet static pressure, measured } \\ \text { NCC } & \text { Compressor spool speed, measured } \\ \text { T3 } & \text { Combustor inlet total temperature, measured } \\ \text { P8 } & \text { Combustor inlet static pressure, measured } \\ \text { NPT } & \text { Low-pressure turbine spool speed, measured } \\ \text { P10 } & \text { Fuel static pressure, actuator signal } \\ \text { TT } & \text { Demanded torque, actuator signal } \\ \text { BV1 } & \text { Bleed valve position } \\ \text { BV2 } & \text { Bleed valve position } \\ \text { IGN } & \text { Ignition, actuator signal } \\ \eta & \text { isentropic efficiency } \\ \vartheta & \text { mass flow } \\ A & \text { cross section area } \\ \varphi & \text { angle } \\ \omega & \text { angular velocity } \\ \lambda & \text { relative air/fuel ratio } \\ h & \text { enthalpy } \\ n & \text { number of mole } \\ m & \text { mass } \\ m_{f l o w} & \text { mass flow } \\ X & \text { concentration vector }\end{array}$

\section{ACKNOWLEDGMENT}

The research has been funded by the Swedish Energy Agency, Siemens Industrial Turbomachinery AB and Volvo Aero Corporation through the Swedish research program TURBO POWER, the support of which is gratefully acknowledged.

A special thanks is also addressed to Mats Sjödin at Siemens Industrial Turbomachinery in Finspång, Sweden.

\section{REFERENCES}

[1] Volponi, A. J., 2003. Foundation of Gas Path Analysis (Part $I$ and II). von Karman Institute Lecture Series: Gas Turbine Condition Monitoring and Fault Diagnosis, January.

[2] Borguet, S., and Léonard, O., 2008. "A sensor-fault-tolerant diagnosis tool based on a quadratic programming approach". Journal of Engineering for Gas Turbines and Power, 130(2), p. 021605.

[3] Kobayashi, T., and Simon, D. L., 2005. "Evaluation of an enhanced bank of kalman filters for in-flight aircraft engine sensor fault diagnostics". Journal of Engineering for Gas Turbines and Power, 127(3), pp. 497-504.

[4] Association, M., 2005. Modelica - a unified object-oriented language for physical systems modeling, language specification. http: / / www. modelica.org.

[5] Idebrant, A., and Näs, L., 2003. "Gas Turbine Applications using Thermofluid". In Proceedings of the 3rd International
Modelica Conference, P. Fritzson, ed., The Modelica Association, pp. 359-366.

[6] Wahlström, J., Eriksson, L., and Nielsen, L., 2010. "EGRVGT control and tuning for pumping work minimization and emission control". IEEE Transactions on Control Systems Technology.

[7] Jankovic, M., and Kolmanovsky, I., 2000. "Constructive lyapunov control design for turbocharged diesel engines". IEEE Transactions on Control Systems Technology.

[8] Heywood, J. B., 1992. Internal Combustion Engine Fundamentals. McGraw-Hill series in mechanical engineering. McGraw-Hill.

[9] McBride, B. J., Zehe, M. J., and Gordon, S., 2002. NASA Glenn Coefficients for Calculating Thermodynamic Properties of Individual Species. NASA/TP-2002-211556.

[10] Kobayashi, T., and Simon, D. L., 2007. "Hybrid kalman filter approach for aircraft engine in-flight diagnostics: Sensor fault detection case". Journal of Engineering for Gas Turbines and Power, 129(3), pp. 746-754.

[11] Frisk, E., Krysander, M., and Åslund, J., 2009. "Sensor placement for fault isolation in linear differential-algebraic systems". Automatica, 45(2), pp. 364-371.

[12] Krysander, M., and Frisk, E., 2008. "Sensor placement for fault diagnosis". IEEE Transactions on Systems, Man, and Cybernetics - Part A: Systems and Humans, 38(6), pp. 1398-1410.

[13] Krysander, M., Åslund, J., and Nyberg, M., 2008. “An efficient algorithm for finding minimal over-constrained subsystems for model-based diagnosis". IEEE Transactions on Systems, Man, and Cybernetics - Part A: Systems and Humans, 38(1).

[14] Blanke, M., Kinnaert, M., Lunze, J., and Staroswiecki, M., 2003. Diagnosis and Fault-Tolerant Control. Springer.

[15] Merrill, W., DeLaat, J., and Bruton, W., 1988. "Advanced detection, isolation, and accommodation of sensor failures - real-time evaluation". Journal of Guidance, Control, and Dynamics, 11, pp. 517-526.

[16] Menke, T., and Maybeck, P., 1995. "Sensor/actuator failure detection in the vista $\mathrm{f}-16$ by multiple model adaptive estimation". IEEE Transactions on Aerospace and Electronic Systems, 31, pp. 1218-1229.

[17] Kobayashi, T., and Simon, D. L., 2003. Application of a Bank of Kalman Filters for Aircraft Engine Fault Diagnostics. NASA/TM-2003-212526.

[18] Page, E., 1954. "Continuous inspection schemes". Biometrika, 41, pp. 100-115.

[19] Hairer, E., Norsett, S. P., and Wanner, G., 1991. Solving Ordinary Differential Equations II: Stiff and DifferentialAlgebraic Problems, 2nd revised edition ed. Springer, Berlin.

[20] Pantelides, C. C., 1988. "The consistent initialization of differential-algebraic systems”. SIAM J. Sci. Stat. Comput., 9(2), pp. 213-231. 